\title{
How the Arrangement of Content and Location Impact the Use of Multiple Distributed Public Displays
}

\author{
Jorgos Coenen, Eslam Nofal, Andrew Vande Moere \\ Research[x]Design - Department of Architecture - KU Leuven, Belgium \\ \{jorgos.coenen, eslam.nofal, andrew.vandemoere\}@kuleuven.be
}

\begin{abstract}
Novel social, civic or entertainment opportunities might emerge when spatially distributed public displays become interlinked in meaningful ways. Yet little is still known about the effect of intrinsic design dimensions, such as how multiple displays should be spatially arranged, how their content should be linked, and how their locations and content should dynamically change over time. We therefore conducted a two-month long design study of a distributed public display system that invited passers-by to answer hyperlocal questions. By comparing the performance of different content and location arrangement strategies, we reveal distinct spatiotemporal user engagement patterns, and the specific local conditions that shaped them. We also discovered several contextual factors that inhibit more widescale engagement, among which the conceptual novelty, the apparent purpose, and the perceived cumulative effort to engage with several displays. Consequently, this study provides insights on how public displays can be linked to augment the effects of distribution.
\end{abstract}

\section{Author Keywords}

Smart cities; public displays; location-based services; Internet of Things; geocaching; civic participation; urban interaction design; community engagement; civic engagement; distributed displays; networked displays; embedded visualization.

\section{cSS Concepts}

- Human-centered computing Field studies • Humancentered computing Empirical studies in ubiquitous and mobile computing • Human-centered computing $\mathrm{HCI}$ theory, concepts and models

\section{INTRODUCTION}

Different kinds of visual media continue to proliferate our urban space, particularly in high traffic areas where multiple screens are sharing the same physical environment. Most of these public displays are controlled by a centralized system that synchronizes a predefined set of playlists or animations. Consequently, these displays convey either identical content to achieve a more consistent, noticeable or overwhelming

Permission to make digital or hard copies of all or part of this work for personal or classroom use is granted without fee provided that copies are not made or distributed for profit or commercial advantage and that copies bear this notice and the full citation on the first page. Copyrights for components of this work owned by others than the author(s) must be honored. Abstracting with credit is permitted. To copy otherwise, or republish, to post on servers or to redistribute to lists, requires prior specific permission and/or a fee. Request permissions from Permissions@acm.org.

DIS '19, June 23-28, 2019, San Diego, CA, USA

(C) 2019 Copyright is held by the owner/author(s). Publication rights licensed to

ACM.

ACM 978-1-4503-5850-7/19/06 ..\$15.00

https://doi.org/10.1145/3322276.3322294

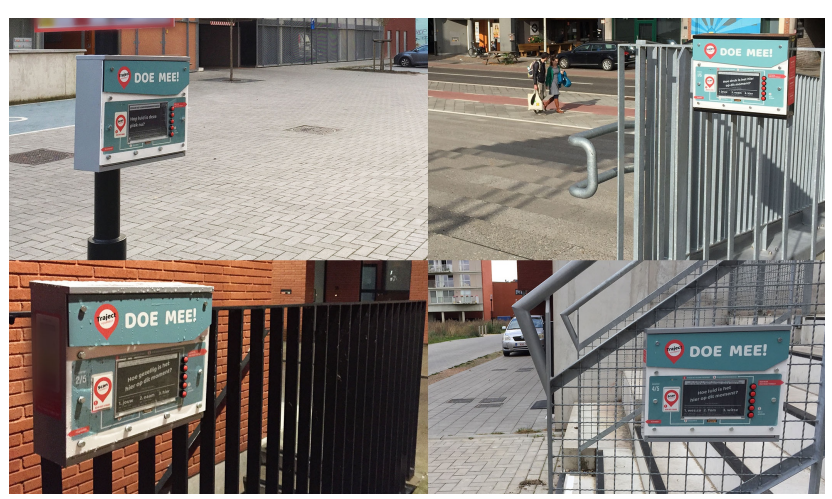

Figure 1: Checkpoints mounted at various contexts within the neighborhood. All content is in Dutch.

visual experience, or each autonomously displays unique content, resulting in a more scattered and unorganized overall visual effect. We call such public display clusters 'distributed' when they are spatially dispersed on an architectural (e.g. [5]) or urban scale (e.g. [19]). Consequently, and in contrast to chains or constellations of co-located screens, distributed displays do not appear as a single installation (e.g. [24]) and require meaningful 'linking' to become seen as part of the same system [33].

The spatial distribution of displays is typically motivated out of a desire to offer a broader amount of potential points of interaction to a large audience, or to reach a wider range of social demographics $[12,28]$. Yet it seems apparent that new commercial, civic or entertainment applications might emerge when the linking of distributed public displays becomes purposeful or meaningful. Novel application domains that have been evaluated so far include the use of multiple displays to visualize and encourage personal physical exercise [7], to convey location-based narratives in a university building [38], to provide wayfinding directions in a busy train station by way of dynamic animations that are split over multiple consecutive displays [5], to reveal invisible contextual information such as local histories to passers-by at a pub [13], or to convey real-time data and comments that relate to a civic issue like local air pollution [2]. However, little is known about the impact on user engagement of diverse design dimensions that intrinsically relate to distributedness, such as how multiple displays should be spatially arranged, how their content should be linked, and whether and how these locations and contents should dynamically change over time.

We therefore conducted an exploratory design study in order to compare the impact of different arrangement strategies in 
terms of how to locate multiple public displays in space, and how to determine the content they convey. First, we customdeveloped an interactive, battery-powered and wirelessly networked public display system that allowed us to update its content periodically and capture user polling, without the need for external infrastructural resources (see Figure 1). We deployed this system, which we coined "TrajectYourself", in the context of a situational urban action that encouraged passers-by to provide hyperlocal feedback about their immediate environment. In practice, this system invited passers-by to: 1) opportunistically discover one or more public displays; 2) receive or collect an identification badge and optionally associate some demographic information with it; and 3) capture one's physical presence at different displays by logging with the badge and answering a hyperlocal polling question.

In this paper, we investigate how two spatiotemporal factors influence the engagement of passers-by with multiple, interactive public displays: 1) how they are distributed in space, and 2) how their content is updated over time. We thus report on an in-the-wild evaluation study that captured various types of empirical evidence by observing and electronically logging the user interactions that occurred over a total period of two months (October, November). By comparing the performance of three different content and location arrangement strategies, we reveal distinct spatiotemporal user engagement patterns and the local conditions that impacted these patterns. We also discovered several contextual factors that inhibited more widescale engagement, among which the conceptual novelty, the apparent purpose, and the perceived effort to engage with more than one display based on sightlines and perceived spatial boundaries. Consequently, we believe this study provides valuable insights on how multiple public displays could become 'linked' for useful purposes and application domains in the future.

\section{RELATED WORK}

A display positioned in a (semi-)public space needs to overcome major user engagement inhibitors like interaction blindness [20], the inability to recognize that a screen is interactive; and display blindness [17], the disregard for a screen that is often caused by the general expectation that its content is irrelevant. A public display yet also possesses the intrinsic quality to be spatially situated and opportunistically reach a diverse audience, who might even be digitally illiterate, with contextual information.

\section{Distributing public displays}

Multiple public displays are able to exploit their spatial distribution in several distinct ways, namely by presenting location-specific content at each location (e.g. [13] and [3]); by taking specific advantage of their spatial arrangement (e.g. for wayfinding information [5]); or by creating potential points of engagement for diverse audiences who may visit other locations (e.g. [7]). There exist many examples in which multiple displays have been spatially distributed, ranging from an architectural scale (e.g. CloudDrops [21]), over a neighborhood scale (e.g. DataOnSite [2]), to an entire city (e.g. UBI-Hotspots [20], Screens in the Wild [25]). Similarly, the screen types and sizes of distributed displays range from miniature e-ink screens [2] (approx. 3-inch), over large LCD displays (57-inch) [20] to rather bespoke and pixel-less installations [37] that yet are all able to convey information publicly. Even devices that are typically aimed at personal use may be considered a type of distributed public display. For instance, specifically designed content on smartphones can be observed by people in the same room [14] and the faces of smartwatches are already glanced at by others and can thus be used to communicate timely content specifically to the glancer or the general public [23].

It is known that the amount and quality of interactions provoked by a public display can be optimized by positioning it at the 'sweet spot' between its intended audience, its content and its physical location [26]. This contextual design space has recently been broadened for distributed displays with the concept of 'linking' [33], which encapsulates how potential users can be motivated to discover and perceive multiple displays to belong to the same system. Already proposed linking tactics include keeping a consistent outer aesthetic, and using different types of guidance to the other displays, such as providing playful hints or detailed instructions, to appeal to different user types [33]. Accordingly, it has been suggested that the distance and visibility between displays influences the ability of passersby to discover them [33].

\section{Public polling displays for civic engagement}

Interactive public displays can facilitate the simultaneous gathering and conveying of different types of civic information from, and to, citizens $[11,29]$. As such, studies have proposed the civic potential of public polling displays as a platform for citizens to be seen and heard $[8,12]$, to evoke a sense of community [18], to trigger civic discourses between local passers-by [27], or to act as data-informed conversation starters $[12,30]$. Past experiments have evaluated bespoke, tangible installations that playfully invite passers-by to answer a wide variety of open-ended and closed questions $[9,28]$, over more low-cost and light-weight technologies, such as wirelessly controlled e-ink displays [2] or electronically augmented posters [34]. Initiatives exist that centralize the control of multiple mobile public display devices into a single digital platform, allowing communities to capture and share local knowledge and ideas [13]. Most public polling systems forgo users to identify themselves to lower the perceived barrier of participation, as a result potentially valuable evidence is missing on repetitive or demographic-specific behaviors [28, 34].

\section{Urban Data Visualization}

As a specialized form of public display, urban data visualization aims to augment the meaning of a place by positioning particular data within a physical context where it is relevant [31], often its original location of measurement. By situating or embedding [35] the data in an interpretable, physical context, passers-by are encouraged to reflect on the behaviors that caused particular patterns, mainly around 
locally relevant or contested topics [2]. Here, data is treated as a boundary object that allows citizens to familiarize themselves about sensor-evidenced issues with personal relevance [29,32]. Often a form of public polling is integrated to trigger curiosity and as a data source that in and by itself sparks discussion [11,12].

\section{TRAJECT YOURSELF}

TrajectYourself is a distributed public display system we custom-developed after being co-awarded a small municipal grant from the city of Leuven, a mid-sized city in Belgium. Their open call aimed to fund about 10 small, experimental initiatives that use technology to encourage people to be more "physical active in the city". Although the municipality was not directly involved in the project, their approval proved beneficial in acquiring many required permits.

Via TrajectYourself, we aimed to reveal some yet unexplored design aspects that influence how people interact with a distributed public display system. We were particularly interested in enticing people to interact with multiple displays when these are scattered around different locations. Therefore, we framed TrajectYourself as a temporary urban intervention that gathered the hyperlocal perceptions of passers-by about public space, such as about when and how specific locations are being used, who occupies them, and how they are subjectively perceived (e.g. noise, temperature, safety). In order to encourage sustained participation, the system was meant to track each meaningful polling interaction, and award individual participants with points that could be accumulated over the period of one week. This simple gamification technique intended to encourage more sustained and even collaborative forms of engagement, as points were valorized as motivational sparks in different ways: to be awarded a 'mayor' title of a specific location (e.g. similar to Foursquare's Swarm), to become listed in a competitive ranking that was shown on the displays (see Figure, top left), or to jointly collaborate to reach a larger, shared point reward towards a good cause.

Consequently, low-cost technological methods had to be tested to track the presence of individual participants at a display in anonymous, yet quick and intuitive ways. After learning that Bluetooth-driven identification via smartphones required either a separate registration process at each display or the activation of a dedicated smartphone application or website, we chose to provide custom RFID key fobs to participants to identify themselves, hereby also allowing fully anonymous participation if deemed necessary.

\section{Registration Portal}

Passers-by became aware of TrajectYourself through various triggers [36], such as by reading promotional flyers that were left at local venues (see Figure ) or distributed on the street, by encountering a physical system component in the environment itself, or by perceiving other participants. A central portal, depicted in Figure 2, acted as an eye-catching promotion platform. Its location was clearly depicted on all promotional materials and shown on the side of each display. Embedded within the portal, a touch-enabled LCD display invited passers-by to take a RFID-equipped badge and

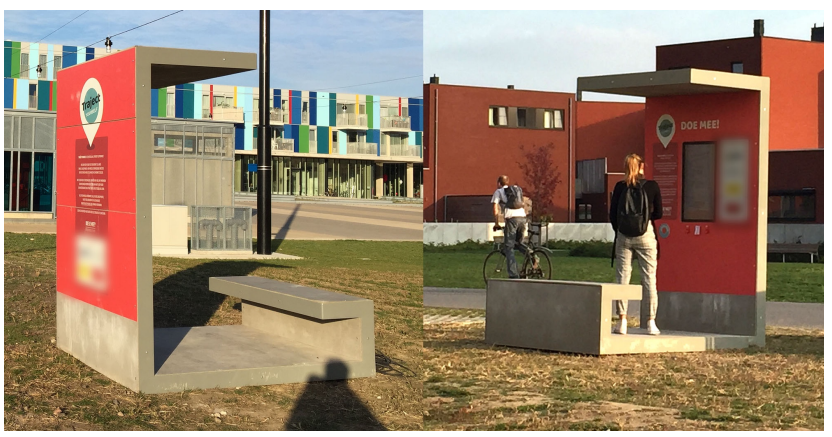

Figure 2: The portal includes seating to encourage leisurely use. Here it is situated along the main pathway.

register it. This process consisted of answering basic demographic questions, including user name, gender, age range, an email address and a reason for visiting the location (i.e. living locally, shopping or commuting). The validity of the answers was not checked to allow for anonymous participation, and creative user names or false email addresses. Each question included a "prefer not to say" or equivalent option. Although solely checkpoint interactions with badges were rewarded with points, it was not required to link the badges with (valid) registrations.

\section{Public Display Checkpoints}

After registering, participants were invited to discover public displays in the immediate vicinity (see Figure 1), answer the questions that were shown on them, and receive individual points as a reward. We deliberately called the distributed public displays 'checkpoints' to reflect that they could be intentionally discovered or be regularly 'checked' within existing daily routines. Participants could retrieve all checkpoint locations via a map that was shown on the portal display or via printed maps at the portal that could be taken along.

Each checkpoint was equipped with an RFID scanner and five push buttons, allowing people to quickly and opportunistically respond to Likert-type multiple-choice questions. If a button was pushed without first scanning a badge, the display showed instructions about where to find the portal. If a button was pushed after scanning a badge, a confirmation message was displayed inviting the participant to return at a later time or to look for other checkpoints nearby, along with a bar chart or bubble map of the historical polling results (see translated display screenshots, Figure ).

\section{Technology}

The portal was constructed out of wood paneling layered with an epoxy substrate and mounted around a custom metal frame, as shown in Figure 2. It housed a Raspberry Pi 3B+ to control the integrated RFID-scanner and 32" touchenabled LCD display. The portal was networked through a commonly available mobile $3 \mathrm{G}$ WIFI router. Custom red vinyl stickering along all sides described some basic participation instructions in a recognizable and consistently branded manner. The badges were commercially-bought passive MIFARE RFID key fobs transmitting at $13.56 \mathrm{MHz}$, which could be detected by the MF-RC522 sensors 


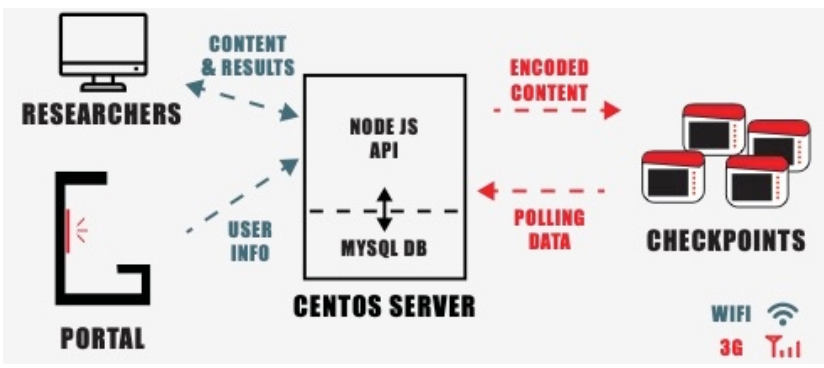

Figure 1: This technical overview of TrajectYourself depicts the bidirectional networking between the portal, the checkpoints, and the off-site, centralized back-end system.

integrated into the portal and the checkpoints. We chose to authenticate users via a custom RFID-badge instead of via the Bluetooth protocol on their personal smartphones in order to avoid requesting that passers-by download a dedicated application, or indeed own a smartphone.

The 7.5" monochrome e-ink display and 3G-enabled STM32F205 ARM Cortex M3 microcontroller of the TrajectYourself checkpoints are based on our open source Citizen Dialogue Kit project [4]. E-ink technology was chosen for its low-energy usage, and its superb readability during even extreme ambient light conditions. It should be noted that our relatively large e-ink display requires approximately 6 seconds to fully refresh, during which flickering occurs. At the start of the flickering, however, the new content becomes already visible. All electronic equipment was powered by a single rechargeable USBconnected $8000 \mathrm{mAh}$ battery pack which allowed each checkpoint to run for approximately one to two days without maintenance. Moreover, this setup allowed us to deploy the checkpoints freely at any location independently of power sources or WIFI access.

A CentOS server functioned as our back-end system and ran a custom Node JS API, which was used to schedule the display content and retrieve the polling results (see Figure 1). All graphic content was converted into an e-ink display compatible encoding, and 'pushed' to the checkpoints in a prescheduled way. Vice versa, each checkpoint locally logged the user interactions and pushed these into a MySQL database on the server in an hourly rhythm, so as to conserve the power required for wireless networking. Upon registration, the portal pushed the user info to the MySQL database on the server.

We dedicated considerable attention to making the physical construction affordable, yet weather and vandalismresistant. Each display was housed inside a standard, aluminum mailbox, as we exploited its rainproof and lockable door mechanism to make the battery easily accessible. Additional layers of laser cut Perspex and colorful vinyl stickers hid the mailbox appearance, physically framed the exposed interface elements, and covered the most obvious points of (violent) entry, as depicted in Figure 1. Consequently, we were able to limit the total financial cost of a single checkpoint to approximate 200 Euro (230 USD). Over the whole study duration, only one

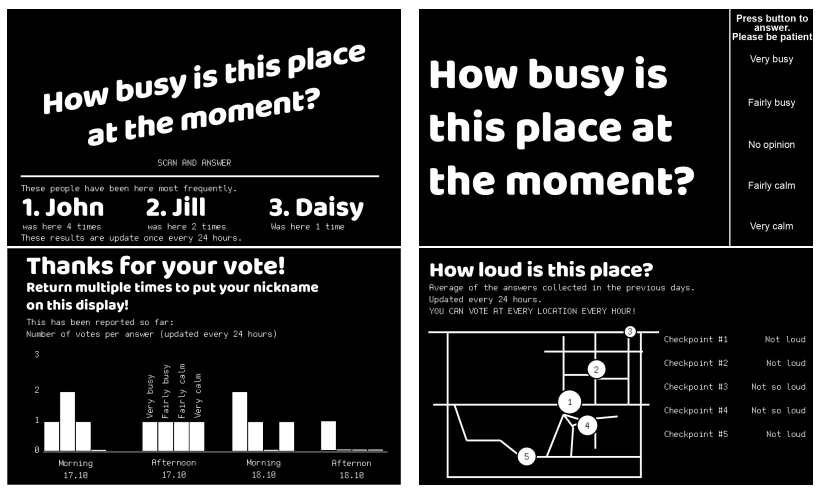

Figure 4: Screenshots of the checkpoint interface translated from Dutch. Top left: a starting screen featuring the current 'mayors' ranking. Top right: the polling question. Bottom left and bottom right: two different visualizations of the polling results so far, shown immediately after the polling action.
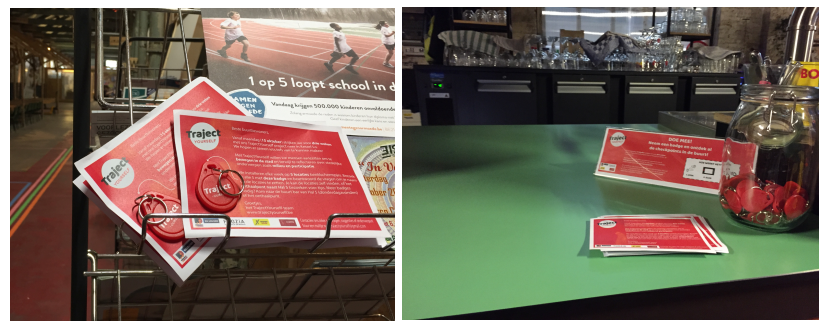

Figure 5: Flyers and badges distributed at the community hub. checkpoint was unexpectedly removed and opened. Surprisingly, all its parts were later recovered unharmed, except of the USB battery pack.

\section{METHODOLOGY}

\section{Pilot Study}

We conducted the pilot test inside a small park close to the city center, a location that was prebooked due to strict yet time-consuming regulatory requirements. During the testing period of three weeks, we identified and addressed various issues in relation to interaction and display blindness, and debugged a wide range of technical issues. To gauge the basic effectiveness of the system, badge registrations were obligatory, and all checkpoints were scattered throughout the cityscape, requiring some effort to find and reach them. Moreover, we increased the relative distance between checkpoints each successive week, so that the connecting route grew from $700 \mathrm{~m}$ to $1 \mathrm{~km}$ and $1.2 \mathrm{~km}$ respectively.

A total of 106 interactions was recorded with 39 badges, of which half occurred via the portal. During three days and at five locations, a total of 40 brief in-situ interviews were conducted. We noticed that as the distance between the checkpoints increased, the participation rate decreased, particularly outside of the park perimeter. Accordingly, we hypothesized that potential users estimate the required physical effort by a perceivable zone of opportunistic play. As we found the obligatory registration requirement too restricting, registrations were made optional during all subsequent deployments. Polling required a badge, yet the badge did not have to be registered at the portal. 


\begin{tabular}{|c|c|c|c|c|c|}
\hline \multicolumn{2}{|c|}{ Conditions } & \multicolumn{4}{|c|}{ Arrangement Strategies } \\
\hline Name & Duration & Location & Location Update & Content & Content Update \\
\hline Roaming & 4 weeks & $\frac{\text { clustered }}{\text { in space }}$ & $\begin{array}{l}\text { locations change } \\
\text { weekly }\end{array}$ & $\begin{array}{l}\text { competitive ranking } \\
\text { based on number of } \\
\text { personal check-ins }\end{array}$ & $\begin{array}{l}\text { polling questions change } \\
\text { weekly for all checkpoints }\end{array}$ \\
\hline Linear & 2 weeks & $\begin{array}{l}\text { linearly } \\
\begin{array}{c}\text { positioned along a } \\
\text { road }\end{array}\end{array}$ & $\begin{array}{l}\text { locations stay } \\
\text { unchanged }\end{array}$ & $\begin{array}{l}\text { cooperative financial } \\
\text { support of a good cause }\end{array}$ & $\begin{array}{l}\text { polling questions rotate } \\
\text { daily between checknoints }\end{array}$ \\
\hline Scattered & 2 weeks & $\frac{\text { clustered }}{\text { in space }}$ & & check-ins & \\
\hline
\end{tabular}

Table 1: Summary of explored arrangement strategies and their distinct combinations inside the conditions. Note that the Roaming condition is twice as long as the other two conditions due to the weekly location updates.

\section{Study Context}

All the TrajectYourself infrastructure was transferred to just outside of the city center, in order to evaluate its performance in the context of a quiet neighborhood instead of the busy pace of a city center. Located next to the back entrance of a busy train station, the neighborhood surrounds a relatively heavily trafficked walking and cycling pathway and is depicted in Figure . Being relatively newly developed, the housing is mostly occupied by young families and people from various (international) backgrounds. A derelict train maintenance building has been re-appropriated to host community-led social and commercial initiatives, such as a social grocery shop, an organic bakery and a coffee roastery. Opened only in the afternoon from Wednesday to Sunday, this building functions as an informal community hub.

At various occasions, all indicated in Table 2, flyers and badges were handed out to passers-by along the main cycling and pedestrian path. Badges and flyers were also made continuously available at the portal, the neighborhood café and the social grocery store. The content of all checkpoint displays was updated daily around 10am to reflect the polling results from the previous day.

\section{Arrangement Strategies}

The study setup was divided into three distinct conditions, listed in Table 1. Each condition combines a location and content arrangement and their dynamic alteration over days or weeks, called an update.

Location. The clustered arrangement strategy exemplifies how each display could be independently located in a unique spatial context, requiring people to scavenge them. The linear arrangement puts multiple displays closeby along a road, so that the effort required to discover and use them is lowered. The weekly update of the clustered arrangement aimed to test how the mobility of a public display could tap into more and smaller zones of local interest, which however requires repetitive efforts to rediscover them.

Content. Similarly, the displays' content either featured a competitive motivation strategy, showing the top-ranking user names according to their number of check-ins; or a cooperative strategy, showing the total monetary award for a good cause that was gained by aggregating all check-ins of the past week together. In Scattered and Linear, we promised to donate 25 eurocents (0.30 USD) and up to 150 Euro per week to our university hospital's Child Cancer Fund for each

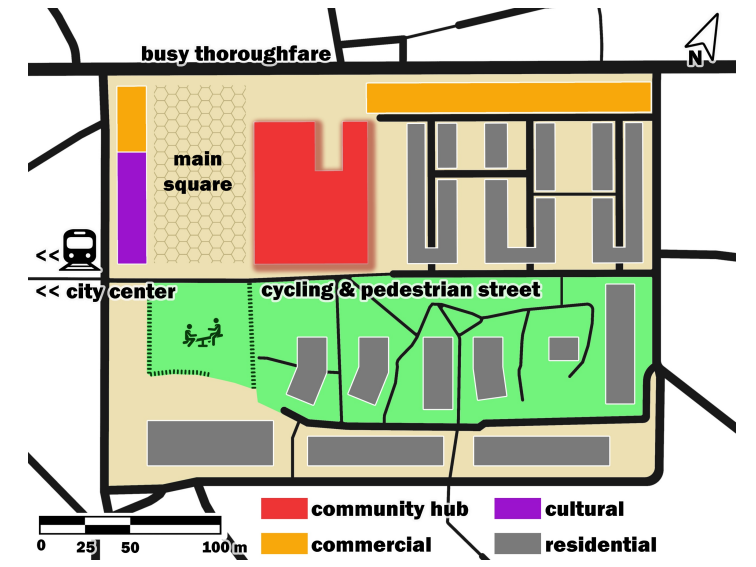

Figure 6: Map of the setting with markings indicating the main streets, major building types and grassy areas.

check-in that occurred with a badge. Each badge could be used to check-in at every checkpoint once every hour, and the competitive leaderboard that showed the top-ranking participants was replaced by a progress bar showing the total of collected funds. As a result, we donated 65 Euro (74 USD) during Linear and 32,50 Euro (37 USD) during Scattered.

The content update arrangement indicates how polling questions were either updated weekly in bulk for all checkpoints at once, or rotated daily across the displays, in an attempt to reveal whether content refreshing impacts participation.

The portal was located on the border of a large grass square, alongside the cycling and pedestrian pathway, and in close vicinity of the community hub (marked as central registration point or CRP in row 1 of Table 2). In the third week of Roaming, due to unexpected regulatory requirements, the portal had to be moved approximately 50 meters along the pathway to the terrace right in front of the community hub. The approximate locations of the checkpoints can be observed in the first row of Table 2.

\section{Data Collection}

All interactions, such as check-ins, registrations and portal visits, were digitally logged. Due to a complex hardware issue, all the logged data of checkpoint \#3 during condition Roaming was deemed untrustworthy and was not included in our analysis. Yet we consider all data collected from the remaining checkpoints valid and worthy of inclusion. The 


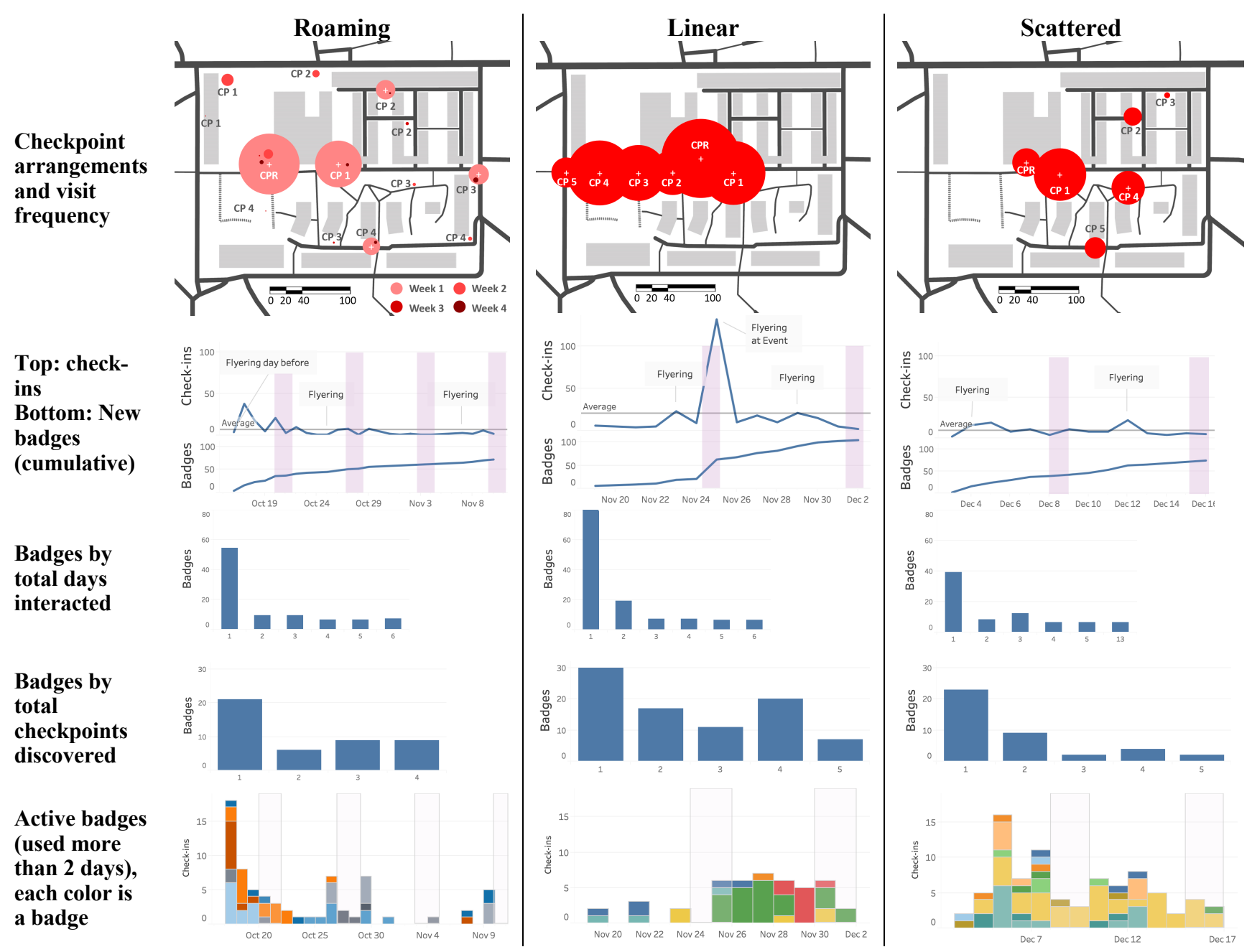

Table 2: Graphical summary of the results. Weekends are marked by light blue boxes where applicable. Dot size encodes frequency of visits in row 1 .

qualitative results rely on in-situ occasional observations, informal run-ins with participants and semi-structured interviews. In addition, we invited the 63 participants who registered their badge, provided a valid email address and indicated that they could be contacted for more in-depth interviews in exchange for an 8 Euro gift certificate. As a result, we conducted a total of 41 interviews in-situ (20 with more than one person, $<5$ minutes), and 10 interviews via phone or email ( $\sim 20$ minutes), between 10 and 20 days after the study, depending on their availability. All Dutch interview quotes were translated by the authors for inclusion in this paper. The logging data was analyzed statistically and via exploring various data visualizations. The interview data was analyzed through several thematic analyses by the primary researcher.

\section{RESULTS}

Table 3 summarizes the overall quantitative results from the three conditions. When offsetting the larger number of discovered checkpoints in the Linear condition which are partly due to a popular social event close by, we consider Scattered to show the most sustained public engagement.
Hyperlocal polling. Participants considered the hyperlocal polling functionality to be interesting and relevant, exemplified by one participant who appreciated "especially the question about noisiness ... because this is a real concern for people in the neighborhood". While all participants remembered multiple polling questions during the post-hoc interview, they did not remember well the results that were visualized immediately after their interactions (bottom row of Figure ). At most, positive or negative tendencies, or alignments with one's own opinion was remembered. One person mentioned that she "wasn't sure if these were [her] results, or a summary" because the graph aligned with her own submitted opinion. As we also observed, the relatively slow refresh rate of the e-ink displays seems to be the most obvious reason, as recurrent participants optimized their interaction flow: "the first time I read everything, but then I knew how it worked and didn't have to wait for the display to fully update each time." For some, the hyperlocal polling became the core motivation, as they appreciated its citizen science aspect and felt empowered to convey their 'personal expertise' on a topic (i.e. the space) about which they felt confident (e.g. "I enjoy the idea that I can have a voice [...], 


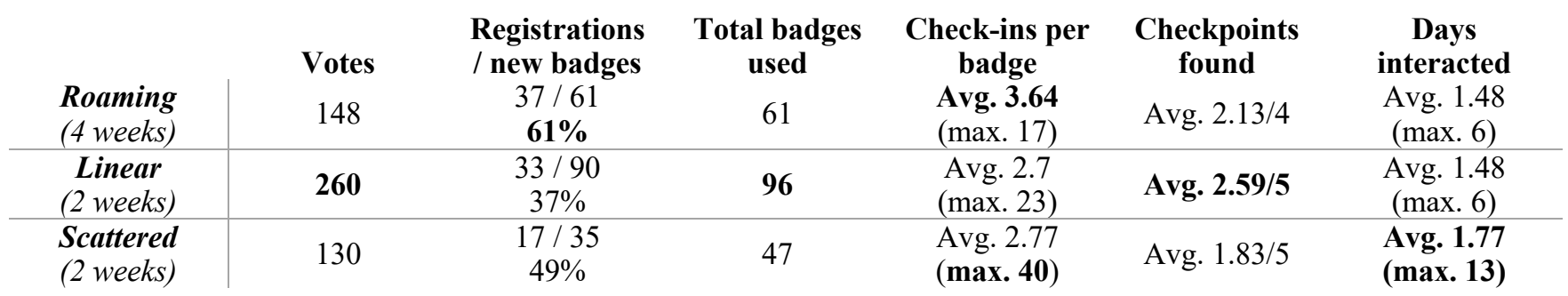

Table 3: Overview of quantitative results of the three different conditions. The largest values are highlighted in bold.

We consider the usage of badges a proxy for user participation, however multiple people may share a single badge.

questions to me as a local 'expert'."). Conversely, some people felt less inclined to submit votes for those locations that they were not "familiar with or actually pass often", as they felt no stake in potentially improving them. One participant, herself a non-native Dutch speaker, expressed concerns about the language, referring to the mixed background of the inhabitants, "It's a diverse neighborhood, if you want a full perspective, you need to have English".

Hyperlocal polling seems like a potentially trustworthy application domain. In Linear, when the checkpoints were only approximately 30 meters apart, both the reported "coziness" and "cleanliness" ratings decrease from the safe community hub towards the checkpoint where the cycling road intersects with regular car traffic (see Figure). Interestingly, the area between these two locations was reported to be "unsafe" and "loud", which is likely due to that this area is used by teenagers to socialize and play music.

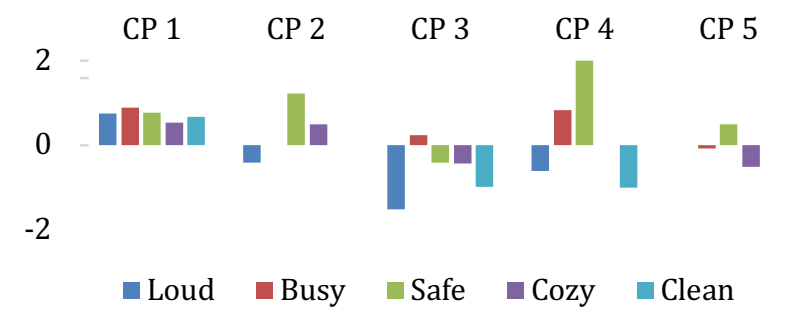

Figure 7: Hyperlocal polling results ranging from very positive (2) to neutral (0) and very negative (-2) for the different checkpoints (CP1-5) during the Linear condition.

Display technology. While the e-ink display technology was mentioned as being novel and readable, it was also perceived as slightly disconcerting. Participants are unfamiliar with eink technology and were surprised by the latency and amount of flickering during content refreshes, e.g. "The display was a little strange the first time. It started to flicker, and I was not sure if I did it right.".

Participation recruitment. It took considerable time for most ( 8 out of 10) of our in-depth interviewed participants to become active for the first time after having initially noticed the displays. This period took even weeks for at least 5 participants. In contrast, most people interviewed in-situ were well aware of the multiple checkpoints (21/24). Everyone had noticed the checkpoints during their regular, daily habits, e.g. "... I therefore quickly noticed something new". Yet this awareness seemed inefficient in triggering passers-by to engage. Instead, manual recruitment strategies proved more effective, evidenced by how the participation peaks coincide with the flyering events (marked by annotations in row 2 of Table 2). To better understand the impact of recruitment, we compared the performance of 53 badges that were originally attached to flyers that were handed out, versus 82 badges that were taken at the portal, during the three final weeks of the study. Badges originating from flyering were used more often (avg. 1.7 vs 2 check-ins per badge). Yet when portal registrations are disregarded as interactions, a total of $9(32 \%)$ of the badges acquired at the portal were eventually not voted with, versus only $1(7 \%)$ of those during flyering. We believe that people realized the underlying good cause more attentively via the flyers than through the messaging on the public display: "later, when I got a flyer and was told you could support a good cause and that the displays would travel around, I started using it". One non-registered participant who was inactive during Roaming, voted a record 63 times across the two conditions with the good cause, mainly at night (i.e. after 10PM).

Registration. The requirement to use a badge was perceived as cumbersome. Perhaps surprisingly, we found that many passers-by were well aware they needed a badge to participate, knew roughly where to find them, but felt insufficiently motivated to break their walking routine and actively retrieve one. A total of 186 badges were used, of which 87 were registered at the portal. 32 badges (37\%) were registered, yet not used for polling. We suspect that some of these registrations can be attributed to children experimenting with the portal, as we occasionally observed this, and some entered meaningless nicknames (e.g. "ieeeee" or "elephaantttt"). The average self-reported age was 32.5, with more female participants (42), than males (34) and who preferred not to say (11). The majority lived in the local municipality (51), out of which 35 voted and 12 on multiple days. Only 4 out of 21 non-local registered badges were used

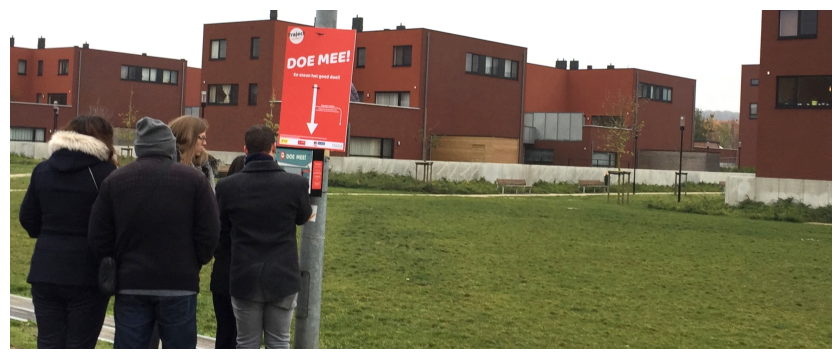

Figure 8: Multiple people sharing one badge. The sign reads (in Dutch): "Participate! Support the good cause." 
during multiple days. We observed regularly how people shared a single badge and interacted in groups (see Figure ).

Discovering multiple displays. The distribution of displays did not succeed in opportunistically changing the walking routines of passers-by. While some interviewees made limited attempts to discover more locations by bringing a map or taking a one-time detour, most acknowledged that they only interacted if and when "they were on my path, I did it multiple times a day. " Participants who deviated from their normal routes already had no immediate goal: "if we go walk the dog, it doesn't really matter where we go". Others were motivated by the underlying good cause, or by experiencing the 'scavenger hunt' aspect together with their children "it's a good excuse to take the children outside when we bike around on Wednesday and weekends", and then expressed interest in making even longer trajectories, e.g. "It could be a whole activity, with a clear route, something fun to do and to discover the city" in a more focused context, e.g. "maybe inside a botanical garden ...".

Exploratory engagement patterns. As shown in Figure 9, journeys along multiple checkpoints on a single day typically commenced at the checkpoint that was closest to the portal (i.e. checkpoint 1), which became perhaps an unexpected inhibitor towards truly dispersed and decentralized forms of user engagement. Frequent engagements typically occurred along four patterns (see Figure 10). Inspecting occurred when people tested out features at one or two checkpoints but

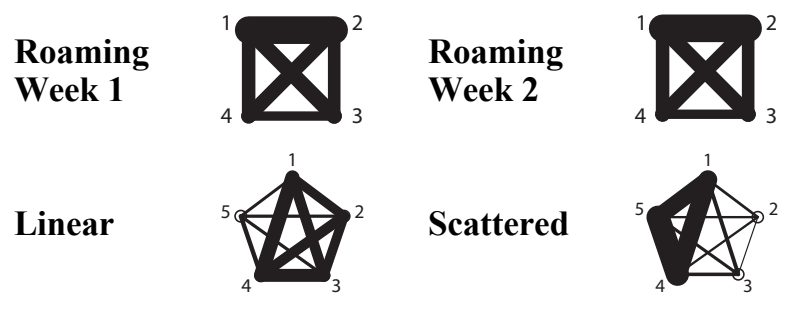

Figure 9: Flow diagrams indicating the pairwise connectedness based on the relative frequency with which checkpoints are visited by the same person(s) on the same day. Checkpoint one is consistently a prominent connection.

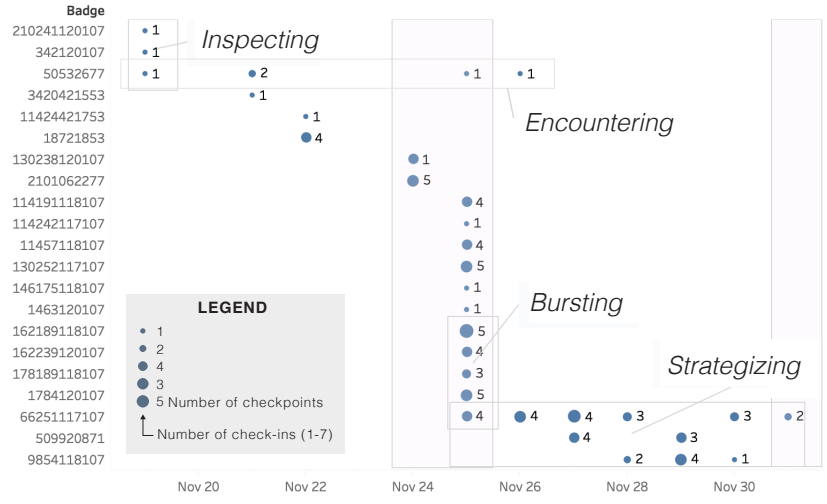

Figure 10: The engagement patterns of a sample of 20 badges (each badge is one row) over time (each day is one column, weekends are light blue). As the embedded legend shows, dot size encodes frequency and numbers the checkpoints visited. Particular engagement patterns are annotated by boxes. feel unconvinced to participate further. Bursting when people interacted intensively in a short period of time, typically during recruitments or other local events. Encountering occurred when people interacted sporadically and perhaps serendipitously. Strategizing points towards more regular and organized occurrences, suggesting a more conscious and goal-oriented kind of motivation.

\section{Roaming Condition}

The spatial clustering of the checkpoints caused some of the further away checkpoints, such as checkpoints 2 in week 1 and checkpoints 1 and 2 in week 2 , to become part of preexisting daily routines, as checkpoints along locally-used pathways received more morning time usage (i.e. before 12PM): "..they were on my way from where I park my car". Yet the location updates caused the overall participation rate to decrease, as some people were confused as to whether, why or when the displays were moved, then suspected they were removed, and did not initiate a search. Some people mentioned how they coincidentally saw the map at the end of the voting process and realize that the checkpoints had not been removed. Most newly acquired participants only interacted for a few days, and most longer-term participants $(5, n=6)$ tended to engage rarely after the update, hereby 'solving' the scavenger hunt. The slight participation uptick in the final week was caused by the checkpoints returning to their initial and thus more trafficked locations (see line graph in Table 2).

We observed some competitive behaviors around checkpoints 1 , mainly between 3 participants in the first week (Figure ). One participant felt triggered by the competitive gaming aspect, and specifically aimed to put the name of his local business at the top of the rankings as a "fun piece of advertising".

10

Nov 17 Nov 18 Nov 19 Nov 20 Nov $21 \quad$ Nov 22

Figure 11: Three participants (score depicted by a line) competing for the first place at checkpoint 1 during Roaming.

\section{Linear Condition}

The linear arrangement strategy caused more and diverse checkpoints to be visited, as relatively many different badges were used at multiple checkpoints. The straightforward spatial configuration aided discovery, as "they were all neatly in a row, very easy to follow", yet they were "perhaps a little too close together, makes you want to skip some." Remarkably, even when the recruitments occurred near checkpoint 5, it was still the least used checkpoint, which again points towards the existence of a more contextual influence. Supporting the good cause became the most important motivation, yet interviewees always recognized the hyperlocal polling as a meaningful purpose regardless: "I was curious about the opinions of others, but most of all I 
wanted to support the fund", or "it's not too much effort and you support a good cause!" The daily rotation of polling questions did not influence participation significantly. As the questions became repetitive and finding the checkpoints was not challenging, one interviewee grounded her motivation via the interest of her young children, "it's the same questions,...but the children don't mind". Another participant states "I would do it maybe four times as long the questions remain relevant".

\section{Scattered Condition}

Both visibility and distance between checkpoints seem to play a role to entice passers-by to visit multiple checkpoints in a single day, as more visible checkpoints lead to more combined visits on the same day and more visits overall. The higher participation rates of checkpoints 1 and 4 can be related to sightlines towards each other and the main walking path. Figure 9 (bottom right) demonstrates a strong connection between these points, indicating their pairwise connectedness through combined interactions. Checkpoint 5, slightly more obscured by buildings, is visited less (see row 1 of Table 2); whereas checkpoints 2 and 3, further removed and visually obstructed, were visited the least.

\section{DISCUSSION}

Although we believe our participation rate is comparable to previously evaluated similar systems, such as $[2,13,33]$, we acknowledge that the achieved participation is too limited compared to expended efforts. None of the conditions or arrangement strategies particularly stand out, instead we discovered a set of situational and specific issues that were more influential than the evaluated arrangements. We therefore believe that our study frames a set of aspects that do not ensure the immediate or massive uptake of a distributed display application, but instead proposes how such a system could become part of local habits and routines.

The fact that people seem to resist opportunistic actions that require some physical and time effort, overarches many of our results. People feel busy and are purposefully traveling to specific locations, within an often tight time schedule. We are somewhat unsure whether a similar intervention without registration and badges would result in a significantly different participation rate in terms of testing how displays can be linked. Nevertheless, by digitally tracing participation, we believe we can discuss major contextual aspects and types of interaction inhibitors in terms of: motivation, hyperlocal 'expertise' and stake, deployment duration, manual recruitment, as well as sightlines and perceived spatial boundaries.

\section{Participation}

Nearly every interviewed passer-by had previously noticed the distributed, physical presence of the display infrastructure while walking by. As such, the common issue of public display blindness seems to only play a limited role in our results, insofar that our system was indeed noticeable but still unable to overcome the expectations of passers-by to be sufficiently relevant, fun or engaging. In short, Traject Yourself faced another issue that can be connected to most interactive public interventions: while its outer design can overcome the natural expectation of irrelevance and become noticed, it is challenging to convince passers-by to more actively inspect the content, or stop their activity to more consciously reflect or interact with the displays.

Naturally, the concept of distributed public displays is new, and initially raises the curiosity of many passers-by. Those willing to halt and inspect one or more displays quickly encountered the requirement of having to own and use a badge. While most of them understood where these could be acquired, the physical or time effort proved too much. It seems an even longer-term deployment duration might partly overcome this barrier, as it took considerable time for people to actively engage after noticing the system for the first time. However, longer-term studies are hard to deploy and are still largely overlooked in urban interaction design research, despite the fact that some viable application domains would target such deployments and typically take years.

\section{Motivation}

We tested two motivational strategies to overcome participation inhibition. Results show that competitive behaviors hardly occurred, as the 'honor' to be featured on a public display did not weigh up to the expected effort, except for at least the one local's playful advertising purpose. However, for most participants TrajectYourself failed to become 'playful' and not only 'gameful' [15], possibly due to the limited potential for self-expression and improvisation in the design. Partaking in a cooperative action towards a common good had a more positive effect, and even encouraged at least one participant in an exceptional way. Because there was no immediate connection between hyperlocal polling and child cancer research, we believe a good cause specifically carried by the local community might have positively impacted the participation rate.

\section{Hyperlocal Polling}

As an attempt to apply distributed displays beyond advertising or entertainment applications, it appears that distributed displays might be particularly useful for crowdsourcing or citizen science initiatives targeting locally shared and locally relevant goals. The ideas behind hyperlocal polling were considered useful, and people answered the polling questions truthfully, even demonstrating that situational contexts of less than $15 \mathrm{~m}$ can be mirrored in bottom-up opinions. The truthfulness of the polling may be impacted by the requirement of a badge, as this likely eliminates some nonsensical and trial voting, yet also lead to a narrower user base.

Our results indicate that people related their willingness to participate in hyperlocal polling to their individual 'expertise' of a place, and to their personal stake in potentially improving it based on the results. As such, distributed displays can deliver high-quality content for those areas where a communal 'stake' is high.

\section{Content Update}

Participants expressed fatigue when having to repeatedly report about similar contextual aspects at somewhat similar locations. Polling questions could better reflect the unique 
characteristics of the immediate environment or update in order to actively react to time-varying conditions.

\section{Purpose}

Display content was not able to overcome display blindness issues when passers-by had already determined the relevance of their contents. This issue is particularly evident in our longer-term study, as many passers-by formed an opinion early on, and subsequently overlooked the cooperative target that was implemented later on. Conveying prompting and activating information unto, or close to, the displays themselves may appear efficient and logical, yet proved much less effective than manual recruitment methods.

\section{Situational Context}

Most of our participation and polling results are best explained by taking into account hyperlocal spatial aspects, more so than the location and content arrangements we actually tested. For instance, displays near to residential areas show different interaction patterns, are less sequentially connected to the other displays, and were used more frequently in morning and at night. Therefore we propose that in order for a system to align with existing practices in a space, besides allowing implicit and passive interaction [16], distributed displays should also take into account spatial aspects, including discoverability (e.g. typical walking routines, sightlines, physical boundaries) and time-varying conditions (e.g. weather, human activities).

Specifically, our flow diagrams depicting the pairwise connectedness of locations uniquely demonstrates which locations facilitate discoverability and linking. Furthermore, they hint to the effects of the boundaries that people perceive within spaces, for example evidenced by the low connectedness of checkpoint 5 in the Linear condition.

\section{Engagement styles}

The adaptability of distributed displays allows passers-by to take on different personal engagement styles, ranging from targeting a game-like ranking, reaching common social goals, or to find the locations of all displays. Yet most potential users first estimate the expected physical activity and time effort required by interpreting the instructions. Here, a certain balance needs to be found, as we discovered particular spatial arrangements are deemed so easy or granular that displays were skipped, whereas dispersed arrangements were too difficult, particularly when displays were less visible.

Harsh display location updates cause people to question if a system has been removed. This is likely related to expectations about the temporary nature of the installation, which is challenging to balance with an application that required moving public displays. Conversely, if displays are static, content which only shifts between the displays may be perceived as repetitive. As a result, although we aimed for serendipitous behavior to occur, the vast majority 'stuck' to their habitual pathways and daily patterns, unless their activities were already aimless (e.g. walking a dog). Targeting a predefined purpose like an urban game would solve these issues, yet defies exploring the civic opportunities of embedding technologies into people's everyday experiences.

\section{SHORTCOMINGS AND LIMITATIONS}

We realize that due to the longer-term and exploratory nature of this study, entanglement of the researchers with the participants is difficult to entirely prevent. Through daily onsite presence, intermitted personal and email interviews, participants, local inhabitants and stakeholders may become familiar with the researchers and their research interests. In this sense, our methodology is related to action research $[6,10]$ as we aim to acknowledge the role of the investigators in these deployments [1]. Similarly, due to our iterative approach within the same neighborhood, it is possible that some passers-by did not engage at a later stage due to experiences at an earlier stage. This contextual focus also implies a single cultural and social context. For example, our study was regularly closely associated to the very bottom-up, civically and politically engaged community space.

Our quantitative results may be biased by the relatively small number of interactions and the many influences outside of our control (e.g. one broken checkpoint in Roaming). Also, the extrinsic motivation of supporting a good cause may have biased the results of the Linear and Scattered conditions.

Finally, the e-ink display technology potentially impacted our results. However, the specific display size, type and interaction modality may be of limited consequence compared to a clear purpose and alignment with contextual factors, such as those described in this paper. Our results reveal that locals did aptly notice even small changes in their immediate environment and knew how to interact. Consequently, relatively small displays that may disregard typical guidelines (e.g. about size and brightness [22]) could become even more granularly integrated in order to be appropriated into common routines.

\section{CONCLUSIONS}

To the best of our knowledge, this study is the first that evaluates the performance of a system that specifically targets interaction with multiple public displays, spatially distributed over a distance of up to 500 meters and deployed over two months.

Overall, we propose that the linking of multiple, distributed displays should take into consideration hyperlocal, situational characteristics, including spatial aspects like discoverability (e.g. walking routines, sightlines, physical boundaries), time-varying conditions (e.g. weather, activities taking place), relevance (e.g. overall purpose or goals, motivations, tasks to fulfill), and personal preferences (e.g. playfulness, stake in the outcome).

Our results suggest that hyperlocal polling with distributed public displays is a feasible application domain, and that locals can be encouraged by giving them a stake in the results and aligning with their routines.

\section{ACKNOWLEDGEMENTS}

This study was funded by the sports division of the Leuven municipality. We also thank participants, Fugzia and Hal 5. 


\section{REFERENCES}

[1] Barry Brown, Stuart Reeves, and Scott Sherwood. 2011. Into the Wild: Challenges and Opportunities for Field Trial Methods. In Proceedings of the Conference on Human Factors in Computing Systems (CHI'11), 1657-1666. https://doi.org/10.1145/1978942.1979185

[2] Sandy Claes, Jorgos Coenen, and Andrew Vande Moere. 2018. Conveying a Civic Issue Through Data via Spatially Distributed Public Visualization and Polling Displays. In Proceedings of the Nordic Conference on Human-Computer Interaction (NordiCHI'18), 597-608. https://doi.org/10.1145/3240167.3240206

[3] Sandy Claes and Andrew Vande Moere. 2017. The Impact of a Narrative Design Strategy for Information Visualization on a Public Display. In Proceedings of the 2017 Conference on Designing Interactive Systems (DIS'17), 833-838. https://doi.org/10.1145/3064663.3064684

[4] Jorgos Coenen, Maarten Houben, and Andrew Vande Moere. 2019. Citizen Dialogue Kit: Public Polling and Data Visualization Displays for Bottom-Up Citizen Participation. In Proceedings of the Conference Companion Publication on Designing Interactive Systems (DIS'19 Companion). https://doi.org/10.1145/3301019.3325160

[5] Jorgos Coenen, Niels Wouters, and Andrew Vande Moere. 2016. Synchronized Wayfinding on Multiple Consecutively Situated Public Displays. In Proceedings of the International Symposium on Pervasive Displays (PerDis'16), 182-196. https://doi.org/10.1145/2914920.2929906

[6] Peter Dalsgaard. 2010. Research in and Through Design: An Interaction Design Research Approach. In Proceedings of the Conference of the ComputerHuman Interaction Special Interest Group of Australia on Computer-Human Interaction (OZCHI '10), 200203. https://doi.org/10.1145/1952222.1952265

[7] Gil-Castineira Felipe, Fernandez Antia Lopez, Lopez Cristina Bravo, Cid-Vieytes Nerea, Conde-Lagoa David, Costa-Montenegro Enrique, and Gonzalez Francisco Javier Castano. 2011. RunWithUs: A Social Sports Application In The Ubiquitous Oulu Environment, In Proceedings of the International Conference on Mobile and Ubiquitous Multimedia, 195-204. https://doi.org/10.1145/2107596.2107621

[8] Claude Fortin, Kate Hennessy, and Hughes Sweeney. 2014. Roles of an Interactive Media Façade in a Digital Agora. In Proceedings of The International Symposium on Pervasive Displays, 7-12. https://doi.org/10.1145/2611009.2611029

[9] Connie Golsteijn, Sarah Gallacher, Lisa Koeman, Lorna Wall, Sami Andberg, Yvonne Rogers, and Licia Capra. 2015. VoxBox: A Tangible Machine That Gathers Opinions from the Public at Events. In Proceedings of the International Conference on Tangible, Embedded, and Embodied Interaction (TEI '15), 201-208. https://doi.org/10.1145/2677199.2680588
[10] Gillian R. Hayes. 2014. Knowing by Doing: Action Research as an Approach to HCI, In Ways of Knowing in HCI, 49-68. https://doi.org/10.1007/978-1-49390378-8_3

[11] Luke Hespanhol, Martin Tomitsch, Ian McArthur, Joel Fredericks, Ronald Schroeter, and Marcus Foth. 2015. Vote As You Go: Blending Interfaces for Community Engagement into the Urban Space. In Proceedings of the International Conference on Communities and Technologies (C\&T'15), 29-37. https://doi.org/10.1145/2768545.2768553

[12] Lisa Koeman, Vaiva Kalnikaité, and Yvonne Rogers. 2015. "Everyone Is Talking About It!": A Distributed Approach to Urban Voting Technology and Visualisations. In Proceedings of the Conference on Human Factors in Computing Systems (CHI'15), 3127-3136. https://doi.org/10.1145/2702123.2702263

[13] Can Liu, Ben Bengler, Danilo Di Cuia, Katie Seaborn, Giovanna Nunes Vilaza, Sarah Gallacher, Licia Capra, and Yvonne Rogers. 2018. Pinsight: A Novel Way of Creating and Sharing Digital Content Through "Things" in the Wild. In Proceedings of the 2018 Designing Interactive Systems Conference (DIS'18), 1169-1181. https://doi.org/10.1145/3196709.3196782

[14] Andrés Lucero, Jussi Holopainen, and Tero Jokela. 2012. MobiComics: Collaborative Use of Mobile Phones and Large Displays for Public Expression. In Proceedings of the International Conference on Human-computer Interaction with Mobile Devices and Services (MobileHCI'12), 383-392.

https://doi.org/10.1145/2371574.2371634

[15] Andrés Lucero, Evangelos Karapanos, Juha Arrasvuori, and Hannu Korhonen. 2014. Playful or Gameful?: Creating Delightful User Experiences. In Interactions 21, 3: 34-39. https://doi.org/10.1145/2590973

[16] Beatrice Monastero and David K. McGookin. 2018. Traces: Studying a Public Reactive Floor-Projection of Walking Trajectories to Support Social Awareness. In Proceedings of the 2018 Conference on Human Factors in Computing Systems (CHI'18), 487:1487:13. https://doi.org/10.1145/3173574.3174061

[17] Jörg Müller, Dennis Wilmsmann, Juliane Exeler, Markus Buzeck, Albrecht Schmidt, Tim Jay, and Antonio Krüger. 2009. Display Blindness: The Effect of Expectations on Attention towards Digital Signage. In Pervasive Computing, 1-8. http://dx.doi.org/10.1007\%2F978-3-642-01516-8_1

[18] Taylor Nick and Cheverst Keith. 2009. Social Interaction Around a Rural Community Photo Display. Int. J. Hum.-Comput. Stud. 67, 12: 1037-1047. https://doi.org/10.1016/j.ijhcs.2009.07.006

[19] T. Ojala, H. Kukka, T. Lindén, T. Heikkinen, M. Jurmu, S. Hosio, and F. Kruger. 2010. UBI-Hotspot 1.0: Large-Scale Long-Term Deployment of Interactive Public Displays in a City Center. In 2010 International Conference on Internet and Web Applications and Services, 285-294. https://doi.org/10.1109/ICIW.2010.49 
[20] Timo Ojala, Vassilis Kostakos, and Hannu Kukka. 2012. Multipurpose Interactive Public Displays in the Wild: Three Years Later. Computer 45, 5. https://doi.org/10.1109/MC.2012.115

[21] Simon Olberding, Jürgen Steimle, Suranga Nanayakkara, and Pattie Maes. 2015. CloudDrops: Stamp-sized Pervasive Displays for Situated Awareness of Web-based Information. In Proceedings of the International Symposium on Pervasive Displays (PerDis'15), 47-53. https://doi.org/10.1145/2757710.2757718

[22] Callum Parker, Martin Tomitsch, and Judy Kay. 2018. Does the Public Still Look at Public Displays?: A Field Observation of Public Displays in the Wild. Proc. ACM Interact. Mob. Wearable Ubiquitous Technol. 2, 2: 73:1-73:24. https://doi.org/10.1145/3214276

[23] Jennifer Pearson, Simon Robinson, and Matt Jones. 2015. It's About Time: Smartwatches As Public Displays. In Proceedings of the Conference on Human Factors in Computing Systems (CHI'15), 1257-1266. https://doi.org/10.1145/2702123.2702247

[24] Walter Robert, Ten Koppel, Gilles Bailly, Jörg Müller, and Walter Robert. 2012. Chained displays: configurations of public displays can be used to influence actor-, audience-, and passer-by behavior. In Proceedings of the Conference on Human Factors in Computing Systems, 317-326. https://doi.org/10.1145/2207676.2207720

[25] Ava Fatah gen. Schieck, Holger Schnädelbach, Wallis Motta, Moritz Behrens, Steve North, Lei Ye, and Efstathia Kostopoulou. 2014. Screens in the Wild: Exploring the Potential of Networked Urban Screens for Communities and Culture. In Proceedings of The International Symposium on Pervasive Displays (PerDis'14), 166:166-166:167. https://doi.org/10.1145/2611009.2617199

[26] Ronald Schroeter, Marcus Foth, and Christine Satchell. 2012. People, Content, Location: Sweet Spotting Urban Screens for Situated Engagement. In Proceedings of the Designing Interactive Systems Conference (DIS'12), 146-155. https://doi.org/10.1145/2317956.2317980

[27] Fabius Steinberger, Marcus Foth, and Florian Alt. 2014. Vote With Your Feet: Local Community Polling on Urban Screens. In Proceedings of The International Symposium on Pervasive Displays (PerDis'14), 44:44 44:49. https://doi.org/10.1145/2611009.2611015

[28] Nick Taylor, Justin Marshall, Alicia Blum-Ross, John Mills, Jon Rogers, Paul Egglestone, David M. Frohlich, Peter Wright, and Patrick Olivier. 2012. Viewpoint: Empowering Communities with Situated Voting Devices. In Proceedings of the Conference on Human Factors in Computing Systems (CHI'12), 1361-1370. https://doi.org/10.1145/2207676.2208594

[29] Nina Valkanova, Sergi Jorda, Martin Tomitsch, and Andrew Vande Moere. 2013. Reveal-it!: The Impact of a Social Visualization Projection on Public Awareness and Discourse. In Proceedings of the Conference on
Human Factors in Computing Systems (CHI'13), 3461-3470. https://doi.org/10.1145/2470654.2466476

[30] Nina Valkanova, Robert Walter, Andrew Vande Moere, and Jörg Müller. 2014. MyPosition: Sparking Civic Discourse By a Public Interactive Poll Visualization. In Proceedings of the Conference on Computer Supported Cooperative Work \& Social Computing, 1323-1332.

https://doi.org/10.1145/2531602.2531639

[31] Andrew Vande Moere and Dan Hill. 2012. Designing for the Situated and Public Visualization of Urban Data. Journal of Urban Technology 19, 2: 25-46. https://doi.org/10.1080/10630732.2012.698065

[32] Andrew Vande Moere, Martin Tomitsch, Monika Hoinkis, Elmar Trefz, Silje Johansen, and Allison Jones. 2011. Comparative Feedback in the Street: Exposing Residential Energy Consumption on House Façades. In Proceedings of the IFIP TC 13 International Conference on Human-computer Interaction - Volume Part I (INTERACT'11), 470488. Retrieved from http://dl.acm.org/citation.cfm?id=2042053.2042103

[33] Giovanna Nunes Vilaza, Can Liu, Ben Bengler, Licia Capra, and Yvonne Rogers. 2018. Here, This and Next: Evaluating Public Engagement with Multiple, Distributed and Interlinked Devices. In Proceedings of the Nordic Conference on Human-Computer Interaction (NordiCHI'18), 524-536. https://doi.org/10.1145/3240167.3240178

[34] Vasilis Vlachokyriakos, Rob Comber, Karim Ladha, Nick Taylor, Paul Dunphy, Patrick McCorry, and Patrick Olivier. 2014. PosterVote: Expanding the Action Repertoire for Local Political Activism. In Proceedings of the 2014 Conference on Designing Interactive Systems (DIS'14), 795-804. https://doi.org/10.1145/2598510.2598523

[35] W. Willett, Y. Jansen, and P. Dragicevic. 2017. Embedded Data Representations. IEEE Transactions on Visualization and Computer Graphics 23, 1: 461470. https://doi.org/10.1109/TVCG.2016.2598608

[36] Niels Wouters, John Downs, Mitchell Harrop, Travis Cox, Eduardo Oliveira, Sarah Webber, Frank Vetere, and Andrew Vande Moere. 2016. Uncovering the Honeypot Effect: How Audiences Engage with Public Interactive Systems. In Proceedings of the 2016 Conference on Designing Interactive Systems(DIS'16), 5-16. https://doi.org/10.1145/2901790.2901796

[37] Niels Wouters, Jonathan Huyghe, and Andrew Vande Moere. 2014. StreetTalk: Participative Design of Situated Public Displays for Urban Neighborhood Interaction. In Proceedings of the Nordic Conference on Human-Computer Interaction (NordiCHI'14), 747756. https://doi.org/10.1145/2639189.2641211

[38] Xiao Emila Yang and Martin Tomitsch. 2014. Designing Interactions with Pervasive Displays for Location-based Storytelling. In Proceedings of the 26th Australian Computer-Human Interaction Conference on Designing Futures (OzCHI'14), 133136. https://doi.org/10.1145/2686612.2686631 